\title{
Slider Posture Effects on Air Bearing in a Heat-Assisted Magnetic Recording System
}

\author{
Kyaw Sett Myo, ${ }^{1}$ Weidong Zhou, ${ }^{1}$ Xiaoyang Huang, ${ }^{2}$ Shengkai Yu, ${ }^{1}$ and Wei Hua ${ }^{1}$ \\ ${ }^{1}$ Data Storage Institute, Agency for Science, Technology and Research (A*STAR), DSI Building, \\ 5 Engineering Drive 1, Singapore 117608 \\ ${ }^{2}$ School of Mechanical and Aerospace Engineering, Nanyang Technological University, \\ 50 Nanyang Avenue, Singapore 639798 \\ Correspondence should be addressed to Kyaw Sett Myo, myo_kyaw_sett@dsi.a-star.edu.sg
}

Received 3 August 2012; Accepted 28 September 2012

Academic Editor: Jia-Yang Juang

Copyright (๑) 2012 Kyaw Sett Myo et al. This is an open access article distributed under the Creative Commons Attribution License, which permits unrestricted use, distribution, and reproduction in any medium, provided the original work is properly cited.

This paper reports the effects of slider posture on the slider bearing in a heat-assisted magnetic recording (HAMR) system with the direct simulation Monte Carlo (DSMC) method. In this HAMR system, the heat issues on the slider bearings are assumed to be caused by a heated spot on the disk and/or slider body itself at various pitch angles. The simulation results show that with a heated spot on the disk, the air bearing pressure and air bearing force that acted on the slider surface will increase when the pitch angle becomes larger. It is also found that the bearing force increases with the heated spot size and the effects of a heated spot become more obvious at a larger pitch angle. On the other hand, the slider body temperature is observed to have a noticeable effect on air bearing pressure and force. The smaller pitch angle enlarges the tendency of bearing force variations with the slider temperature and makes the slider more sensitive to its temperature changes.

\section{Introduction}

With the increasing demands for large amount of storage nowadays, higher density recording technologies are required in hard disk drive industry. Heat assisted magnetic recording (HAMR) is one of the promising technologies to push the recording areal density of magnetic disk drives towards $10 \mathrm{~Tb} / \mathrm{in}^{2}$ and beyond. An HAMR system requires using a focused laser beam to heat up the media so as to reduce the media coercivity. It makes magnetic writing possible over high anisotropy magnetic media [1]. Performing the laser heating process requires the optimized slider designs integrating with the near field optical system inside for precise heating and recording. The integrated slider with an optical heating device normally requires flying at an extremely low head media spacing, which is only around few nanometers nowadays, to provide a high field at heated spot position for successful recording $[2,3]$. With this spacing allowance, the Knudsen number, which represents the level of rarefaction effect in the flow, will be much larger than 1.0 and the flow in the head disk interface region can no longer be assumed as a continuum one. Some modifications of continuum equations with appropriate slip boundary conditions are usually required.

However, it is very challenging to study the slider air bearing in a HAMR head disk interface with the current modified continuum equations due to the heat transfer issues involved in the HAMR system. Although some previous studies [46] proposed some heat transfer models for addressing such problems, these models cannot be used directly with the air bearing model to study heat effects on the slider air bearing. Therefore, we proposed to use direct Monte Carlo simulation (DSMC) method instead to solve the air bearing problems of HAMR application in our previous paper [7] because DSMC method has been used widely for modeling gaseous flows under rarefied conditions similar to the flow in the head disk interface. In that paper, the applicability of DSMC method in studying the air bearing effects of a HAMR slider was validated and the effects of heated spot size and slider body temperature to the slider air bearing were studied. Recently, Fukui et al. [8] studied the bearing pressure characteristics with a heated spot using both DSMC method 
and the conventional molecular gas-film lubrication (MGL) equation with consideration of thermal creep flow. They observed that the results using both methods are agreeable $[8]$.

In this paper, we will continue our works with the focus on the slider's posture effects on the air bearing in a HAMR system. We will investigate how the heat energy from a heated spot on the disk or high temperature slider body itself could contribute to the air bearing along with the slider posture changes.

\section{DSMC Method and Code Verification}

The DSMC method was introduced by Bird in the early 1970s [9] and it is a particle-based simulation method for rarefied gas flow problems. It uses a stochastic algorithm to calculate the molecule collision probabilities and scattering distributions conforming to kinetic theory. As for highdensity recording, the head media spacing is reduced to few nanometers. Consequently, the flow in the head disk interface has the rarefaction effect. In the current head disk interface, the Knudsen number falls between 1 and 10 . Therefore, the gas flow in the interface is considered as a transition flow or free-molecule flow and DSMC method offers a promising tool for numerical simulation of the interface gas flow.

Similar to what we have studied in our previous paper [7], the slider air bearing problem in DSMC simulations is simplified as a two-dimensional, microchannel flow with a stationary, slightly inclined surface (slider) above a horizontal plane (disk) moving in the $x$-direction with the tangential velocity $U_{b}$, as shown in Figure 1 . The constant ambient temperature of $T_{0}=273 \mathrm{~K}$ and a pressure of $P_{0}=1 \mathrm{~atm}$ are maintained at inflow and outflow boundaries by adaptively adjusting the appropriate molecular velocity of particles at boundaries. Both slider and disk surfaces are assumed as fully accommodated ones and the collision model uses the variable hard sphere (VHS) particles. In order to facilitate the simple computation, argon gas is used in all simulation case studies in this paper because it is a simple monoatomic molecule gas with zero internal degrees of freedom. Besides, since the argon gas has similar mean free path and potential energy as those of the air, the bearing characteristics in argon gas should be close to that in air. Therefore, many previous air bearing studies $[10,11]$ regarded argon gas as a good candidate to replace air and used it in the DSMC simulations.

As for validating the DSMC simulation code, the results from Liu and $\mathrm{Ng}$ [10] were used to compare with the outcomes of our simulation tests. The channel length $(L)$ is set as $5 \mu \mathrm{m}$ and the minimum channel height $\left(H_{o}\right)$ is $50 \mathrm{~nm}$ with disk moving velocity of $U_{b}=25 \mathrm{~m} / \mathrm{s}$. In the validation tests, various pitch angles $(\alpha)$ from 0.004 to $0.016 \mathrm{rad}$ are placed. The comparison of pressure distributions on the slider surface is shown in Figure 2 and it illustrates that they are in good agreement. Furthermore, the generalized lubrication equation solution of Fukui and Kaneko [12] using $\alpha=0.01 \mathrm{rad}$ is included for better evaluation and it also agrees with the DSMC result.

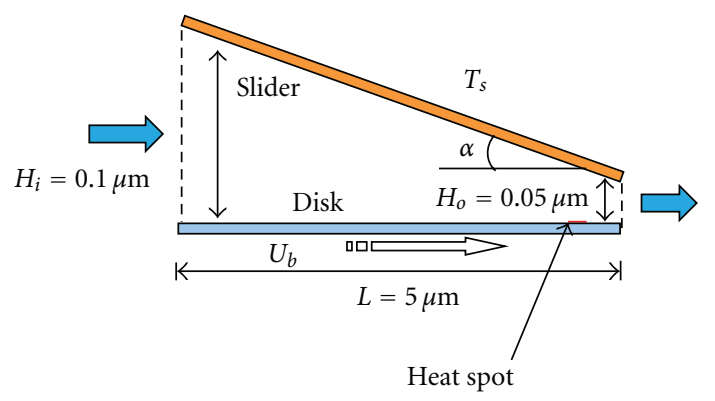

FIgURE 1: The slider and disk layout in the bearing interface.

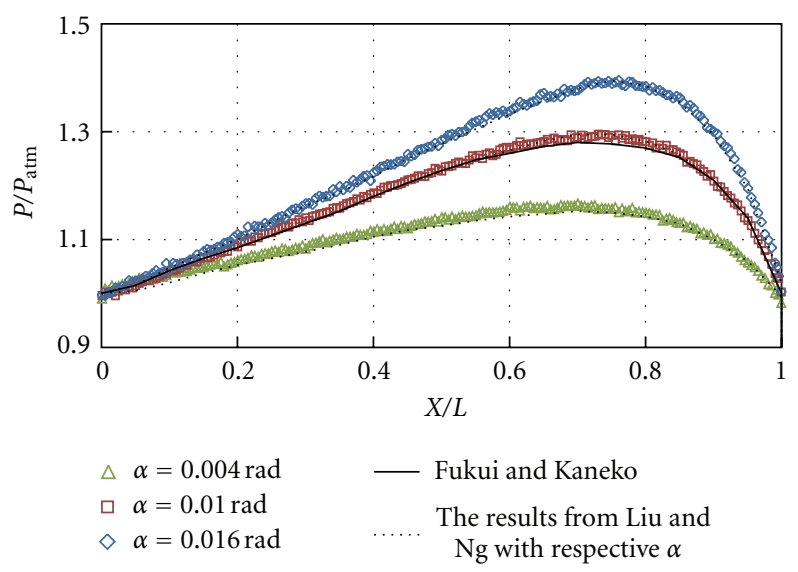

FIGURE 2: Comparison of pressure distributions on the slider surface from the present DSMC code, Liu and $\mathrm{Ng}$ [10] and the generalized lubrication equation solution of Fukui and Kaneko [12].

The streamwise locations of forces with three different pitch angles $(\alpha)$ are compared for validation purpose as shown in Figure 3 and they are also comparable. Here the stream-wise location of the bearing force acted on the slider surface is defined as

$$
X_{c}=\frac{\sum_{i=1}^{n}\left(P_{i}-P_{0}\right) X_{i}}{\sum_{i=1}^{n}\left(P_{i}-P_{0}\right)},
$$

where $n$ is the cell number along the $x$-direction, $X_{i}$ is the individual cell position in $x$-direction, $P_{i}$ is the localized pressure, and $P_{0}$ is the atmospheric pressure.

From Figure 3, we can see that $X_{c}$ is nearly fixed at a constant value when the pitch angle changes. Our DSMC results are also quite comparable with those of Liu and $\mathrm{Ng}$ [10].

After validating our DSMC code, we will use it to study the heat effects caused by a heated spot on the disk or, slider body itself at various pitch angles. The thermal distribution shape of a heated spot on the disk is assumed as a positive half-sine waveform with the peak temperature of $500^{\circ} \mathrm{C}$ $(773 \mathrm{~K})$ as shown in Figure 4 . The slider body temperature $\left(T_{s}\right)$ is assumed to be uniform across the surface of air bearing slider. 


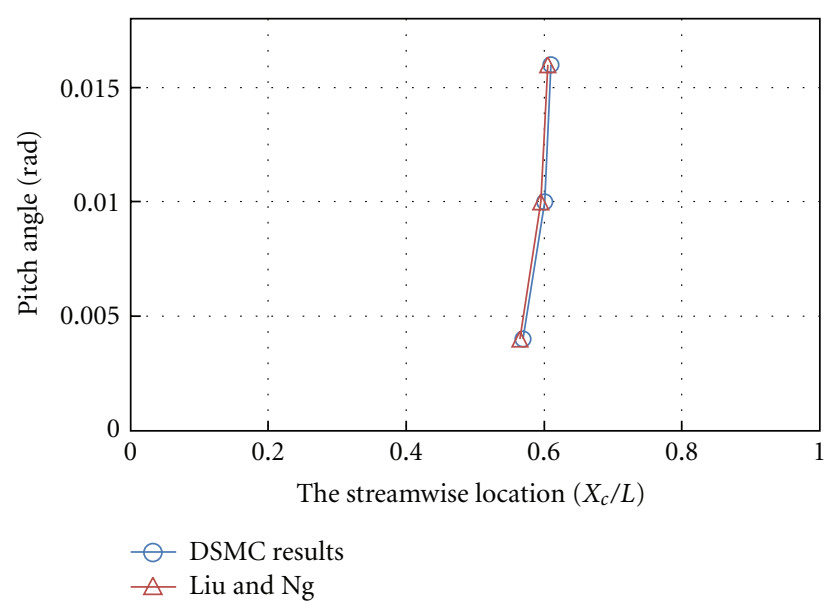

FIgURe 3: Comparison of streamwise locations of the slider bearing forces from the present DSMC code and those of Liu and Ng [10].

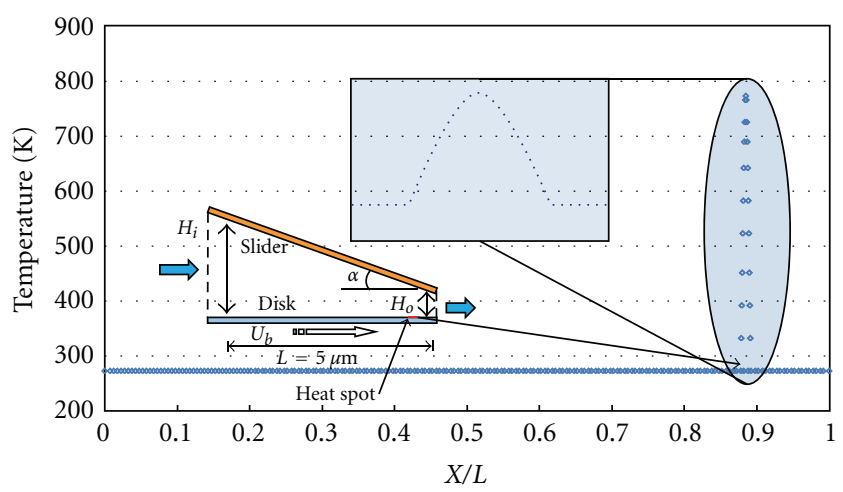

FIGURE 4: The temperature distribution profile with a heated spot on the disk.

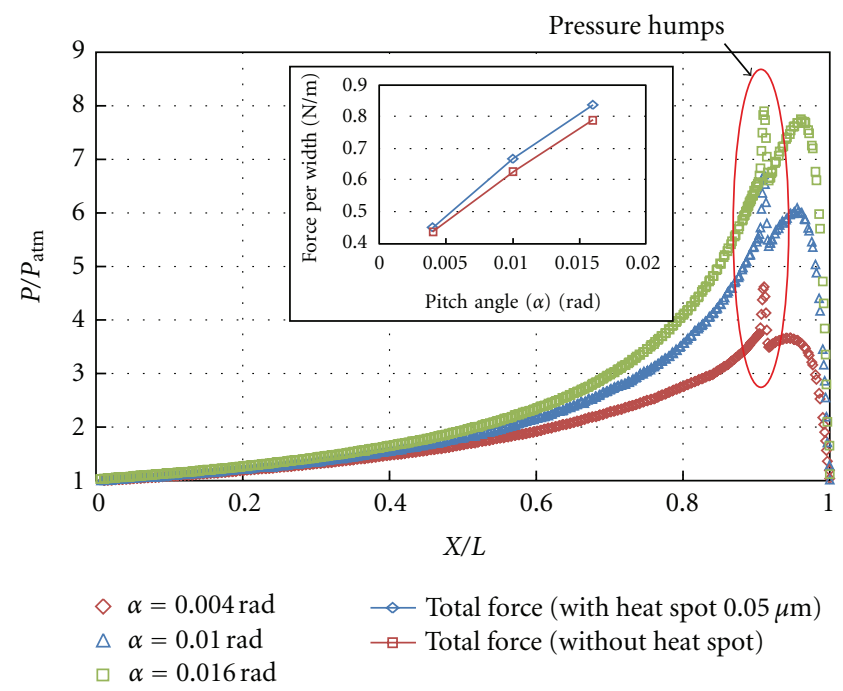

FIGURE 5: Comparison of air bearing pressure profiles on the slider surface with a $0.05 \mu \mathrm{m}$ heated spot on the disk for different pitching angles and (insert) the comparison of bearing forces at different pitch angles with a $0.05 \mu \mathrm{m}$ heated spot and without a heated spot.

\section{Simulation Results and Discussions}

In the simulation tests, the minimum channel height $\left(H_{o}\right)$ is fixed at $5 \mathrm{~nm}$ instead of $50 \mathrm{~nm}$ as for reflecting extremely low head-disk spacing in today's hard disk drives. With the fixed $H_{o}$, the growing pitch angles allow more molecules to come into the interface region. As a result, more molecule collisions happen and the velocities of molecules increase to build up higher pressures acted on the slider surface. The resulted pressure profiles increase with the slider pitch angles as shown in the main diagram of Figure 5.

From our previous study, it was observed that a heated spot on the disk causes the slider bearing pressure and force to increase with its size at the particular pitch angle. However, the heated spot size which is less than $0.05 \mu \mathrm{m}$ does not affect the air bearing substantially [7]. In this paper, the tests were conducted by varying pitch angle $(\alpha)$ from 0.04 to $0.16 \mathrm{rad}$ with the $0.05 \mu \mathrm{m}$ heated spot located on the disk. The heated spot center is situated $0.45 \mu \mathrm{m}$ away from the flow exit in all tests. As in the main diagram of Figure 5, since the overall bearing pressure increases with the larger pitch angle, the amount of pressure hump due to a heated spot is elevated even with the fixed heated spot size. Hence, it assists increasing the bearing force acted on the slider surface.

It is also found that the base area of pressure hump around the heated spot location is wider in small pitch angle than that in the large one. This might be due to the narrower spacing between the slider and disk at a lower pitch, which causes the impact widely from the heated spot to its surrounding. In insert chart of Figure 5, it is observed that the increasing pitch angle enlarges the air bearing force almost in a linear way and the existence of a heated spot increases total bearing force at each posture position. Furthermore, the larger pitch angle causes the higher bearing force on the slider with the same size of a heated spot. Hence, the results suggested that the slider posture affects the air bearing performance with a heated spot which is $0.05 \mu \mathrm{m}$ or larger size in HAMR application.

Figure 6(a) shows the variation of the normalized total force acted on the slider with the heated spot size for different pitch angles. Here we define a normalized force value so that we could evaluate the effect of pitch angle on the total force more clearly. It is found that the variation of normalized bearing force with the heated spot size for a larger pitch angle is higher than that for a smaller one, which means that pitch angle effect becomes more and more important as its value increases. This is because the amount of pressure hump produced by a heated spot, as shown in Figure 5, increases with the pitch angle. Therefore, at a larger pitch angle, the slider bearing force will be more sensitive to the variation of heated spot size.

The stream-wise locations of bearing forces on the slider surface, as shown in Figure 6(b), move closer to the trailing edge as compared with those for larger $H_{o}(50 \mathrm{~nm})$ case. They will shift further towards the trailing edge of slider when a localized heated spot size, that is, larger than $0.05 \mu \mathrm{m}$, is involved in the interface.

Next, as the head disk spacing could be reduced furthermore for higher density recording in HAMR application, it 


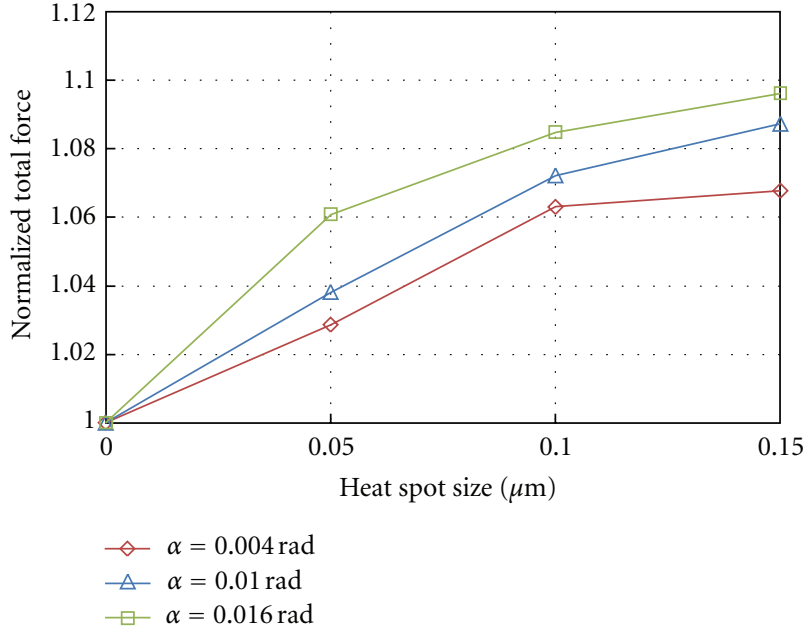

(a)

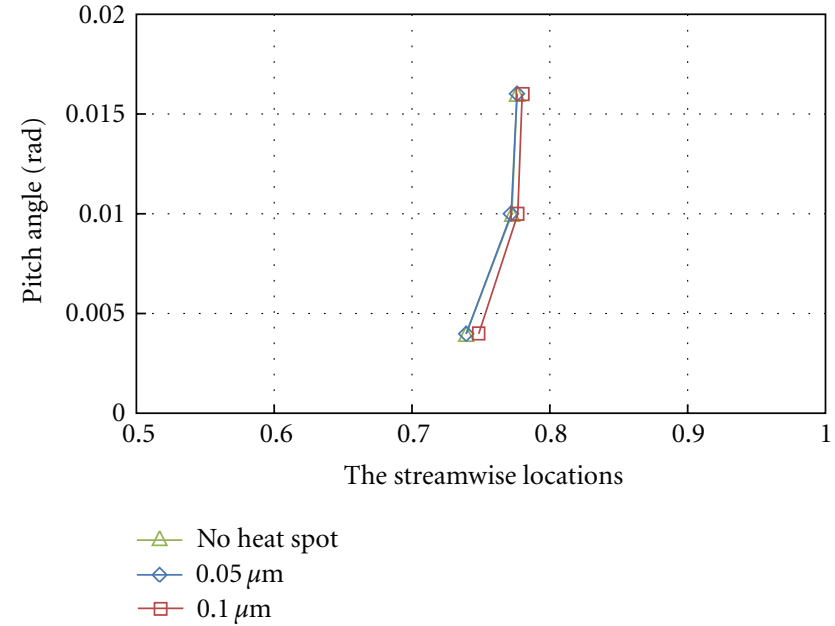

(b)

Figure 6: The variations of (a) total bearing force acted on the slider surface and (b) positions of resultant force with the heated spot size for different pitch angles. The normalized total force represents the respective total force with a heated spot divided by the total force without a heated spot.

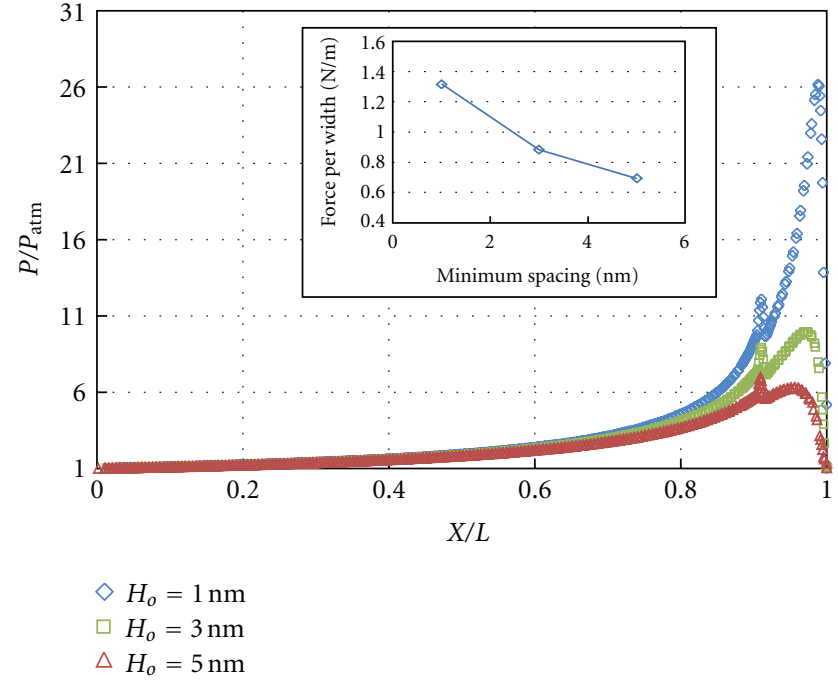

Figure 7: Comparison of air bearing pressure profiles on the slider surface with a $0.05 \mu \mathrm{m}$ heated spot on the disk for different minimum channel heights $\left(H_{o}\right)$ with a fixed pitch angle $(\alpha)$ of $0.01 \mathrm{rad}$ and (insert) the comparison of bearing forces at different minimum channel heights with a $0.05 \mu \mathrm{m}$ heated spot.

is useful to study the effect of smaller gap spacing on the air bearing behavior. In order to study the gap effect, the minimum channel height $\left(H_{0}\right)$ is decreased from $5 \mathrm{~nm}$ to $1 \mathrm{~nm}$ with a fixed pitch angle at $0.01 \mathrm{rad}$. The results are shown in Figure 7. It is found that the bearing pressure increases significantly and its peak position moves away from the pressure bump caused by a heated spot when the minimum spacing $H_{o}$ decreases. From the inset chart of

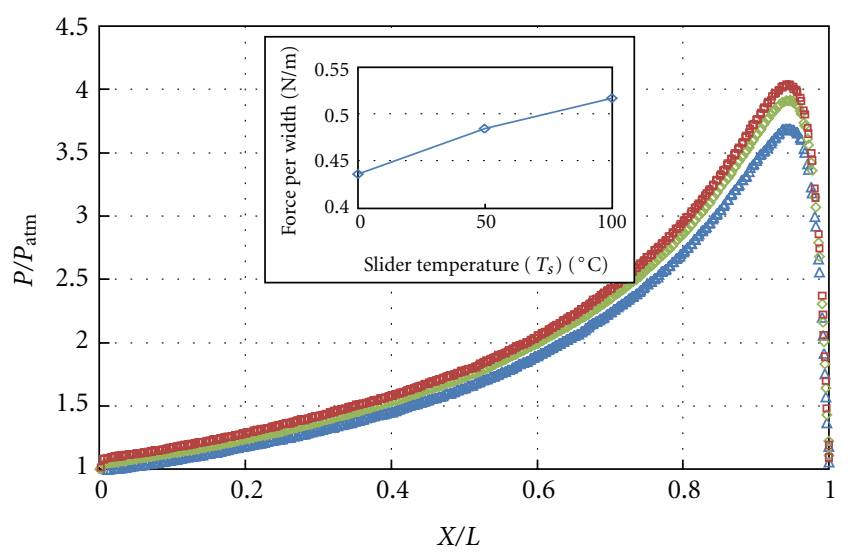
$\triangle T_{s}=0^{\circ} \mathrm{C}$
$\diamond T_{s}=50^{\circ} \mathrm{C}$
ㅁ $T_{s}=100^{\circ} \mathrm{C}$

FIGURE 8: Comparison of air bearing pressure profiles on the slider surface with varied slider body temperatures for a fixed pitch angle $(\alpha)$ of $0.004 \mathrm{rad}$.

Figure 7, it is observed that the bearing force also increases proportionally as $H_{o}$ decreases.

Finally, we study the effect of slider body temperature on the air bearing. We assume that the temperature on the slider surface is higher than the ambient one and it is uniform across the slider surface. Such high temperature on the slider body may be caused by the heat dissipation in the HAMR optical head, electrical heater, writer, or other components. Figure 8 shows that both bearing pressure and force increase gradually as slider body temperature $\left(T_{s}\right)$ changes from $0^{\circ} \mathrm{C}$ to $100^{\circ} \mathrm{C}$ at $\alpha=0.004 \mathrm{rad}$. This is because the thermal energy 


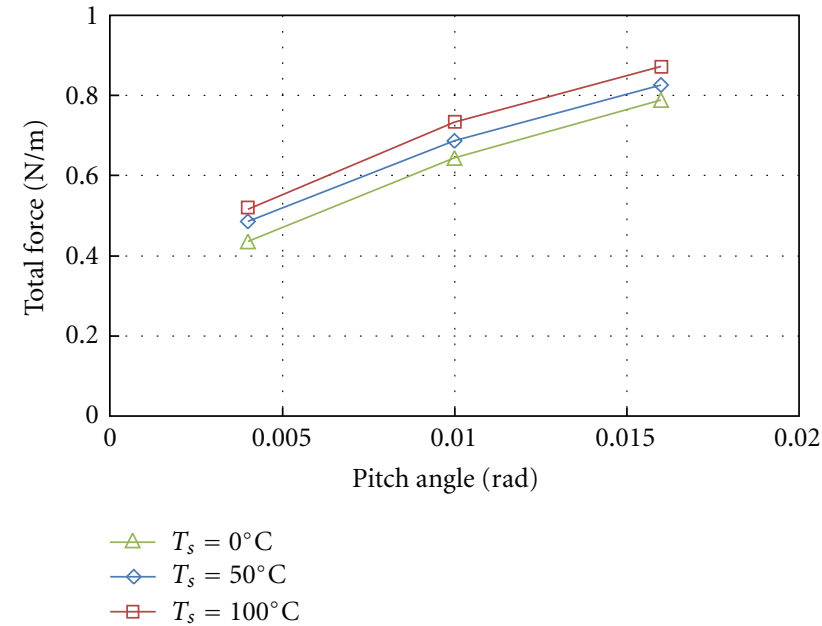

FIgURE 9: Comparison of total bearing forces acted on the slider surface with different slider temperatures and slider pitch angles.

released from the slider body goes into the interface and affects the bearing pressure and force. In other words, the surrounding gas molecules in the interface gain more energy from the heat transfer of a heated spot or slider body and exert stronger pressure on the slider surface when the gas molecules strike on it. As a result, the bearing force, which is derived from the pressure distribution on the slider surface, will increase as the temperature of heated spot or slider body increases. Based on the simulation results with other pitch angles, it is also observed that the total bearing force increments due to slider temperature rises are quite uniform at any slider pitch angle as shown in Figure 9. This suggests that the heat energy from the slider has similar effects on the bearing pressure and force regardless of the pitch angle of the slider.

Figure 10(a) shows the variation of the normalized total force acted on the slider with the slider temperature for different pitch angles. Here we also define a normalized force value to evaluate pitch angle effect at various slider body temperatures. The results show that the normalized bearing force gradient increases with the falling pitch angle, which means that the pitch angle effect becomes less important at its higher values. This is because the total bearing force increment with the slider temperature is almost the same at each pitch angle, but the absolute total force value at $T_{s}=0^{\circ} \mathrm{C}$ will increase with the pitch angle, as shown in Figure 9. Therefore, the normalized force value, which represents the relative change of bearing force at various slider temperatures, will become smaller as pitch angle increases; that is, the air bearing total force is insensitive to the variation of slider temperature at a higher pitch angle.

Figure 10(b) shows the relation of the pitch angle with the slider stream-wise location of force. It is found that the stream-wise location of bearing force shifts towards the leading edge with higher slider temperature. The effect of slider temperature on the location shift is found to be opposite to that of the heated spot.

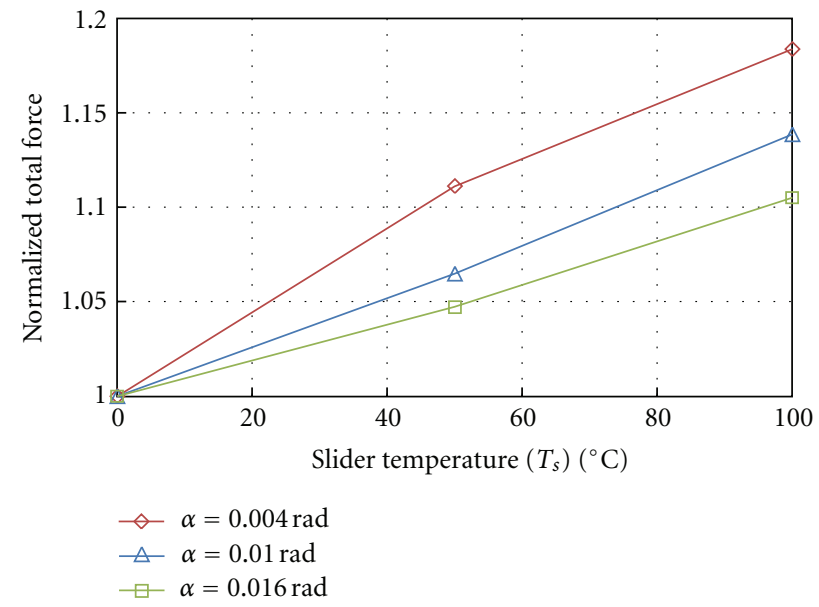

(a)

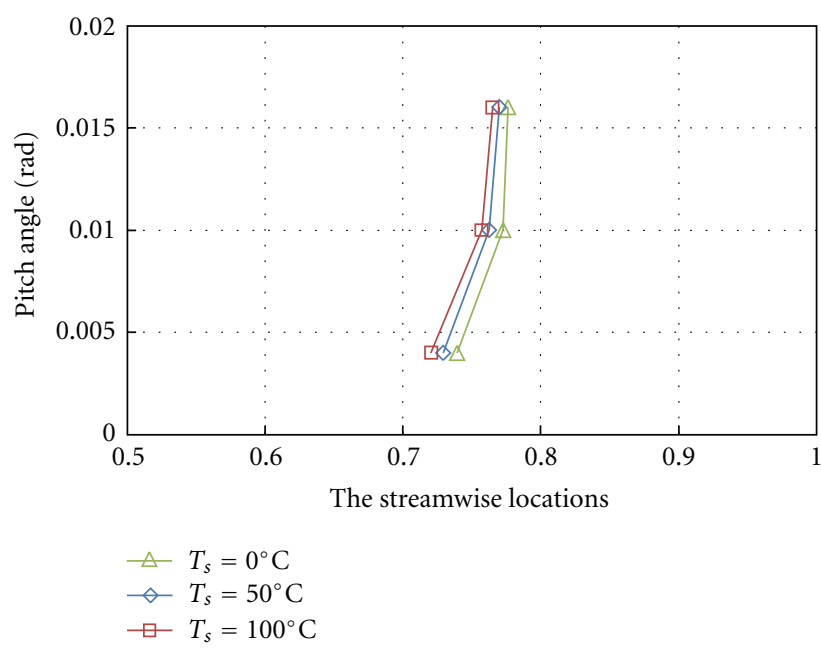

(b)

FIGURE 10: The variations of (a) total bearing force acted on the slider surface and (b) positions of resultant force with varied slider temperatures for different pitch angles. The normalized total force represents the respective total force divided by the total force resulted from the test with $T_{s}=0^{\circ} \mathrm{C}$.

\section{Conclusions}

From the simulation studies in this paper, it is concluded that the slider posture effects on the air bearing should be carefully considered in a HAMR system. The conclusions are drawn as follows.

(1) Both air bearing pressure and total bearing force will increase with the slider pitch angle and the streamwise location of bearing force shifts closer to the trailing edge with the growing pitch angle.

(2) The pressure and force increment caused by a heated spot with a larger pitch angle is higher than that with a lower pitch angle. The slider bearing force will be more sensitive to the variation of heated spot size at a larger pitch angle. 
(3) With a low pitch angle, the effect of a heated spot is small on the air bearing but slider temperature effect will become larger. The slider bearing force will be more sensitive to the variation of slider temperature at a smaller pitch angle.

(4) The stream-wise location of bearing force moves to the trailing edge with a larger heated spot while it goes to the opposite direction as the temperature on the slider body increases.

\section{References}

[1] J. J. M. Ruigrok, R. Coehoorn, S. R. Cumpson, and H. W. Kesteren, "Disk recording beyond $100 \mathrm{~Gb} / \mathrm{in} .2$ : hybrid recording?" Journal of Applied Physics, vol. 87, no. 9, pp. 53985403, 2000.

[2] M. H. Kryder, E. C. Gage, T. W. Mcdaniel et al., "Heat assisted magnetic recording," Proceedings of the IEEE, vol. 96, no. 11, pp. 1810-1835, 2008.

[3] B. Liu, S. Yu, M. Zhang et al., "Air-bearing design towards highly stable head-disk interface at ultralow flying height," IEEE Transactions on Magnetics, vol. 43, no. 2, pp. 715-720, 2007.

[4] W. D. Zhou, B. Liu, S. K. Yu, W. Hua, and C. H. Wong, "A generalized heat transfer model for thin film bearings at headdisk interface," Applied Physics Letters, vol. 92, no. 4, Article ID 043109, 3 pages, 2008.

[5] D. Chen, N. Liu, and D. B. Bogy, "A phenomenological heat transfer model for the molecular gas lubrication system in hard disk drives," Journal of Applied Physics, vol. 105, no. 8, Article ID 084303, 8 pages, 2009.

[6] W. D. Zhou, B. Liu, S. K. Yu, and W. Hua, "Rarefied-gas heat transfer in micro- and nanoscale Couette flows," Physical Review E, vol. 81, no. 1, Article ID 011204, 7 pages, 2010.

[7] K. S. Myo, W. Zhou, S. Yu, and W. Hua, "Direct Monte Carlo simulation of air bearing effects in heat-assisted magnetic recording," Microsystem Technologies, vol. 17, no. 5-7, pp. 903909, 2011.

[8] S. Fukui, N. Kitagawa, R. Wakabayashi, K. Yamane, and H. Matsuoka, "Molecular gas-film lubrication analyses considering boundary temperature distributions," in Proceedings of the Joint International Conference on Micromechantronics for Information and Precision Equipment (MIPE'12), pp. 194-196, Santa Clara, Calif, USA, 2012.

[9] G. A. Bird, Molecular Gas Dynamics and the Direct Simulation of Gas Flows, Clarendon, Oxford, UK, 1994.

[10] N. Liu and E. Y. K. Ng, "The posture effects of a slider air bearing on its performance with a direct simulation Monte Carlo method," Journal of Micromechanics and Microengineering, vol. 11, no. 5, pp. 463-473, 2001.

[11] W. Huang and D. B. Bogy, "Three-dimensional direct simulation Monte Carlo method for slider air bearings," Physics of Fluids, vol. 9, no. 6, pp. 1764-1769, 1997.

[12] S. Fukui and R. Kaneko, "Analysis of ultra-thin gas film lubrication based on linearized Boltzmann equation: first reportderivation of a generalized lubrication equation including thermal creep flow," Journal of Tribology, vol. 110, no. 2, pp. 253-262, 1988. 

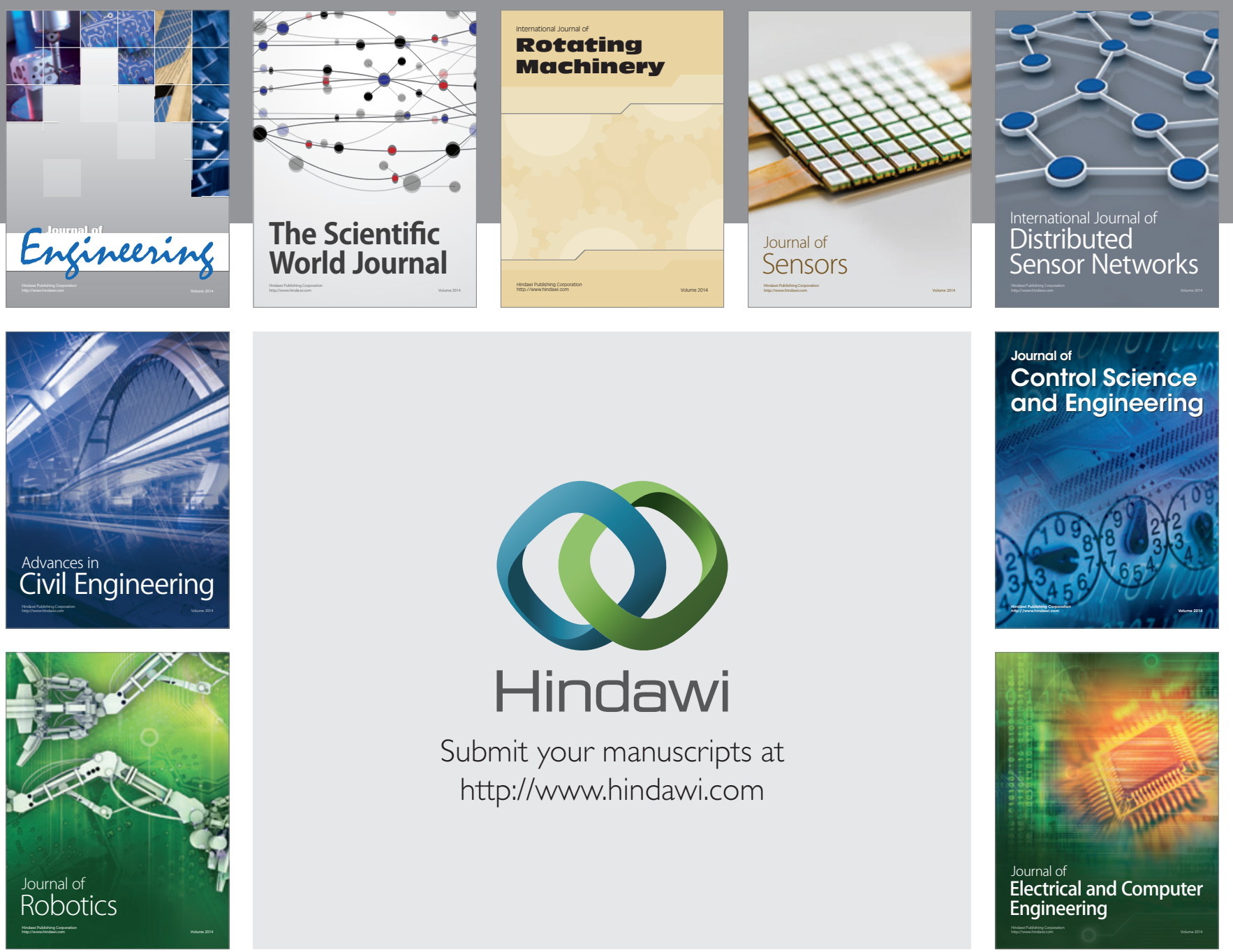

Submit your manuscripts at

http://www.hindawi.com
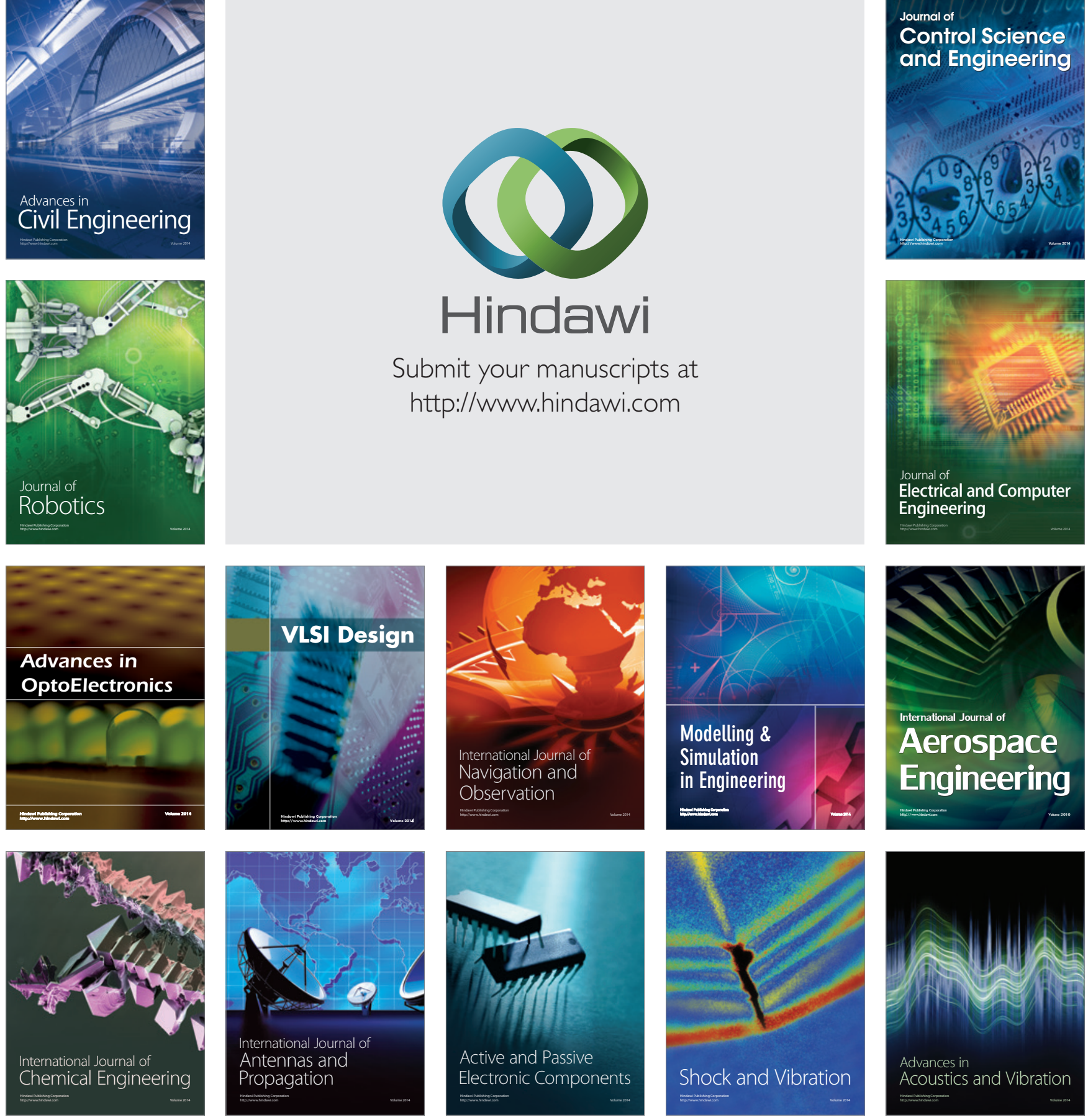